Research Article

\title{
Energy-Efficient Routing Protocol Based on Zone for Heterogeneous Wireless Sensor Networks
}

\author{
Jia Yanfei $\mathbb{D}^{1},{ }^{1}$ Chen Guangda $\mathbb{D}^{1},{ }^{1}$ and Zhao Liquan $\mathbb{D}^{2}$ \\ ${ }^{1}$ College of Electrical and Information Engineering, Beihua University, Jilin 132013, China \\ ${ }^{2}$ College of Electrical Engineering, Northeast Electric Power University, Jilin, China \\ Correspondence should be addressed to Chen Guangda; 694864603@qq.com
}

Received 16 January 2021; Revised 20 April 2021; Accepted 21 May 2021; Published 30 May 2021

Academic Editor: Nicola Pasquino

Copyright (c) 2021 Jia Yanfei et al. This is an open access article distributed under the Creative Commons Attribution License, which permits unrestricted use, distribution, and reproduction in any medium, provided the original work is properly cited.

In heterogeneous wireless sensor networks, sensor nodes are randomly distributed in some regions. In some applications, they may be randomly distributed in different regions. Besides, nodes with the same type have almost the same probability to be selected as cluster head. The cluster head will consume much more energy to receive and transmit data than the other nodes. If nodes with little residual energy have been elected as cluster heads, it will affect the efficiency of the network due to its early death. An improved energy-efficient routing protocol is proposed for heterogeneous wireless sensor networks. Firstly, it supposes that the different types of nodes are distributed in different zones. Secondly, by improving the threshold, nodes with large residual energy have a greater possibility of becoming cluster heads. In the end, it designs a mixed data transmission method. The cluster heads of supper nodes and advance nodes directly transmit data to the base station. The normal nodes adopt single hops and multiple hops mixed methods to transmit data. This can minimize the energy of the communication from cluster head to base station. Simulation results show that this algorithm has achieved a longer lifetime for the wireless sensor network than stable election protocol and threshold-sensitive stable election protocol algorithm.

\section{Introduction}

Heterogeneous Wireless Sensor Networks (HWSN), consisting of many different energy nodes, have become an important focus of research and development $[1,2]$. These sensor nodes are dedicated to sensing environmental events and physical conditions such as floods, fires, and earthquakes. The node compresses the perceived information into data packets, which are sent to the base station (BS) [3-5]. Most of the node's energy is consumed in data transmission. However, as the battery of the nodes is difficult to change after nodes are deployed to the environment, their energy is limited. So this paper mainly discusses the energy heterogeneity of the sensor nodes that have varying levels of energy resources in HWSN.

The stable Election Protocol (TSEP) algorithm [6] is also one of typical Heterogeneous Wireless Sensor Networks. It is supposed that the network contains three different types of sensor nodes that are normal node, advance node, and supernode. The normal node has the lowest initial energy. The supernode has the highest initial energy. The initial energy of the advanced node is between that of the normal node and supernode. It proposed three optimal probability formulas that nodes will be elected as cluster heads without considering the residual energy of the node. For the same type of sensor nodes, although they have different residual energy, they have the same probability to be selected as cluster heads. Therefore, if nodes that have been elected as cluster heads have little residual energy, which will affect the efficiency of the network due to its early death. Besides, the sensor nodes directly transmit data to the cluster head in the same cluster, and the cluster head transmits the received data from other sensor nodes to the based station. The normal node has less energy. When the cluster head of the normal 
node is far away from the base station, if it directly transmits data to the base station, this will consume much energy. This also affects the lifetime of the network. To overcome these problems, we proposed an improved method based on the TSEP method to improve energy efficiency and prolong the network lifetime.

The contributions of the improved TSEP method can be summarized as follows:

(1) To reduce the probability that the sensor node with little residual energy is selected as cluster head, it proposed an improved threshold formula by introducing the residual energy of the sensor node.

(2) To reduce the consumed energy of cluster head, it proposed a combination method of multihop and single-hop for cluster head of normal sensor node transmitting information to the base station.

In Section 1, the introduction is given. In Section 2, we introduce the related work. The TSEP method is introduced in Section 3. In Section 4, we introduce our improved method. The simulation and discussion are given in Section 5. Finally, the conclusions are given in Section 6.

\section{Related Work}

Many effective routing protocols for HWSN have been proposed for achieving a network with a long lifetime and low-energy consumption data acquisition. Stable election protocol (SEP) [7] is a HWSN protocol with two different initial energy nodes. The algorithm delays the death time of the first node because it has two different cluster head $(\mathrm{CH})$ election probabilities. However, the SEP algorithm is only applicable to the two-level HWSN, and the algorithm adopts the method of single-hop routing in data communication. Distributed energy-efficient clustering (DEEC) [8] is designed for multilevel HWSN. It takes the estimated value of the average energy of the node as the reference point and then adjusts the rotation period of the $\mathrm{CH}$ adaptively by the ratio of the reference point and the residual energy.

Numerous heterogeneous protocols based on SEP and DEEC have been proposed. The energy dissipation forecast and clustering management [9] is an improvement of the DEEC method using a "one-step energy consumption forecast" technique. The energy consumption rate based stable election protocol [10] proposes an energy heterogeneous network clustering algorithm based on the node energy consumption rate, which introduces energy consumption rate parameters in the weighted probability of $\mathrm{CH}$ election. The energy proficient clustering technique for lifetime enhancement [11] is a novel energy-efficient distance-based clustering and routing algorithm using a multihop communication approach. Based on distance, the given heterogeneous cognitive radio-based wireless sensor networks are divided into regions and are allocated with a unique spectrum. The zonal-stable election protocol [12] is a clustering algorithm based on zones in which the advanced nodes have the probability of becoming CHs. However, nodes cannot be randomly deployed and only advanced nodes are selected as a cluster. The SEPFL routing protocol based on fuzzy logic [13] is designed by fuzzy logic to elect the optimal cluster. The three fuzzy input variables are the density of nodes, battery energy level, and distance between nodes and BS. Modified stable election protocol [14] is a HWSN algorithm on account of clustering that considers the existence of different transmission types. The energy-efficient prediction clustering algorithm [15] is a hierarchical clustering routing algorithm. All $\mathrm{CHs}$ are selected by the monitored object and the initial energy of the node. The efficient and dynamic clustering scheme for HWSN [16] uses a novel $\mathrm{CH}$ selection method by introducing a universal gravitational law in the clustering process to predict the average network energy. The energy preservation in HWSNs through zone partitioning [17] partitions zones that come through a unified energy efficiency network. It also decreases node communication distance and in selecting the $\mathrm{CHs}$ from their respective zones. The energy efficient clustering scheme for prolonging the lifetime of WSN [18] considers how to deal with the processing problem of the isolated node. The Threshold-Sensitive Stable Election Protocol (TSEP) algorithm [6] is also an improved algorithm based on the SEP method. TSEP algorithm adds supernodes to the network. However, in terms of routing, data is still transmitted to the base station (BS) using the single-hop transmission in TSEP. This routing would consume a lot of energy. The process of the TSEP protocol is described in detail in Section 2. Although some methods have been proposed for mobile sensor nodes $[19,20]$, this paper also still proposed that the sensor nodes cannot be moved in fixed space.

\section{TSEP Method}

The TSEP algorithm, which improved the SEP algorithm, is a heterogeneous wireless sensor network routing algorithm. The TSEP [19] algorithm adds supernodes with more initial energies than other nodes in the new protocol. The TSEP algorithm is divided into two steps: the setup phase and the data transmission phase. The TSEP protocol is described in detail as follows.

In the first phase, the nodes of the wireless sensor nodes are randomly distributed in the HWSN, but the location of each node is fixed. In this phase, each node may be selected as a $\mathrm{CH}$. Each node will be determined by the threshold probability formula to select a $\mathrm{CH}$. The threshold probability proposed in [19] can be expressed as follows:

$$
T(s)= \begin{cases}\frac{p}{1-p[r \times \bmod (1 / p)]}, & \text { if } s \in G, \\ 0, & \text { if } s \notin G,\end{cases}
$$

where $s$ stands the node identifier, $r$ is representative of the current round number, $p$ is the probability that sensor nodes will be elected as $\mathrm{CHs}$, and $G$ represents the node set which has not been selected as a CH. Different sensor nodes with different energy levels have different probabilities to be 
selected as cluster heads. The TSEP algorithm is divided into three types according to the initial energy of the node: super sensor nodes, advanced sensor nodes, and normal sensor nodes, respectively, and their corresponding probabilities that sensor nodes are selected to be cluster heads can be expressed as follows [19]:

$$
\begin{aligned}
P_{\mathrm{nrm}} & =\frac{P_{\mathrm{opt}}}{1+m \times \alpha+b \times \mu}, \\
P_{\mathrm{adv}} & =\frac{P_{\mathrm{opt}}}{1+m \times \alpha+b \times \mu} \times(1+\mu), \\
P_{\text {sup }} & =\frac{P_{\mathrm{opt}}}{1+m \times \alpha+b \times \mu} \times(1+a),
\end{aligned}
$$

where $p_{\text {opt }}$ represents the optimal probability that nodes will be elected as CHs, $m$ and $b$ are the proportion of advance nodes and supernodes to the total number of node respectively, $\mu$ and $\alpha$ is the times that the energy of advance nodes and supernodes is more than that of normal nodes, respectively.

The probability that the sensor node becomes the cluster head is expressed as $P_{\text {opt }}$. In each $1 / P_{\text {opt }}$ round, a sensor node only has one chance to become the cluster head. If a sensor node has not been selected as a cluster head, it will be added into the set $G$, and the process of the cluster head selection will continue in the next round. $P_{\text {opt }}$ is a weight to each node of different initial energy. The weighted probabilities of super sensor nodes, advanced sensor nodes, and normal sensor nodes are expressed as $P_{\text {sup }}, P_{\text {adv }}$, and $P_{\text {nrm }}$, respectively. We use the probability formula (2) to (4) instead of the probability $P$ in (1), respectively. We can get the threshold formulas of sensor nodes, advanced sensor nodes, and normal sensor nodes, respectively. The threshold formulas will be used in the selecting cluster head.

When sensor nodes are selected as cluster heads, they will then broadcast an advertisement (ADV) message by the no persistent carrier-sense multiple access algorithms. After receiving these messages, other non- $\mathrm{CH}$ nodes select a cluster head from cluster heads as their own cluster head according to the signal intensity of each received message. After $\mathrm{CH}$ receive the request message from other nodes, cluster heads will establish a time division multiple access time slot scheduling table. The cluster head then sends the schedule to another sensor node in the same cluster to indicate the presence of the member nodes. When the $\mathrm{CH}$ sends the data, this process can only be completed during a specific time slot and until the formation of the cluster is complete. In the steady phase, data is transmitted from the nodes to the BS, and the data collection process for all the nodes is operated in rounds. The member nodes of clusters transmit information to $\mathrm{CH}$ s based on the time division multiple access slots. Each $\mathrm{CH}$ receives the information, aggregates, and sends the information directly to the BS.
After data transmission is finished in a round, the new $\mathrm{CH}$ selection will be initiated in the next round.

The research results show that the TSEP algorithm has a longer life cycle than the SEP protocol. The TSEP algorithm only prolongs the life cycle of the network by increasing the initial energy of the node. However, there is no fundamental solution to the problem that low-energy nodes consume a large amount of energy and die early.

\section{Proposed Algorithm}

In this section, an improved algorithm is introduced to solve the problem of TSEP. At the setup phase, we place three different initial energy nodes in 3 areas. By improving the threshold formula, the node with larger residual energy has a greater probability of being selected as the $\mathrm{CH}$. We also use a node state transformation mechanism to control the number of $\mathrm{CHs}$ in high-density node areas in Area 2. At the data transmission phase, we adopt single hop and multiple hops to minimize the energy of the communication from $\mathrm{CHs}$ to the BS. We discuss the details of the improved protocol as follows.

4.1. Setup Phase. In the TSEP algorithm, all nodes are randomly deployed in HWSN. All nodes have the potential to be elected as the CHs. The node that has low residual energy may also be elected as the $\mathrm{CH}$. This will reduce the survival time of nodes.

According to the radio energy consumption model proposed in the literature [7], a $\mathrm{CH}$ that is far from the BS to transmit data will quickly deplete more energy. Therefore, the algorithm places supernodes and advanced nodes in an area far from the BS, and other nodes are placed in an area close to the BS. Then we divide the square $M \times M$ into three areas of equal size, and they are called Area 1, Area 2, and Area 3. The BS is located in Area 2. Nodes that are in Area 2 consume less energy than nodes that are in Area 1 and Area 3 as transferring data to the BS. Therefore, we randomly placed 5 supernodes and 10 advanced nodes in Area 1 and Area 3, respectively, and Area 2 is randomly distributed with 70 normal nodes. The distribution of sensor nodes is shown in Figure 1.

After that, the nodes are placed, all nodes in the network broadcast their own information including the node label, the remaining energy of the node, and the node status. The $\mathrm{BS}$ receives messages and stores the node information table including the node label, the remaining energy of the node, and the node status. The normal node receives its own neighbor node messages and stores a neighbor node information table including the node label and the node status. This process is done only once after the node is deployed. Then, we will take two methods to select the $\mathrm{CH}$. Area 1 and Area 3 use one method, while Area 2 uses another method.

Area 1, Area 2, and Area 3 perform $\mathrm{CH}$ selection according to the following equations, respectively, 


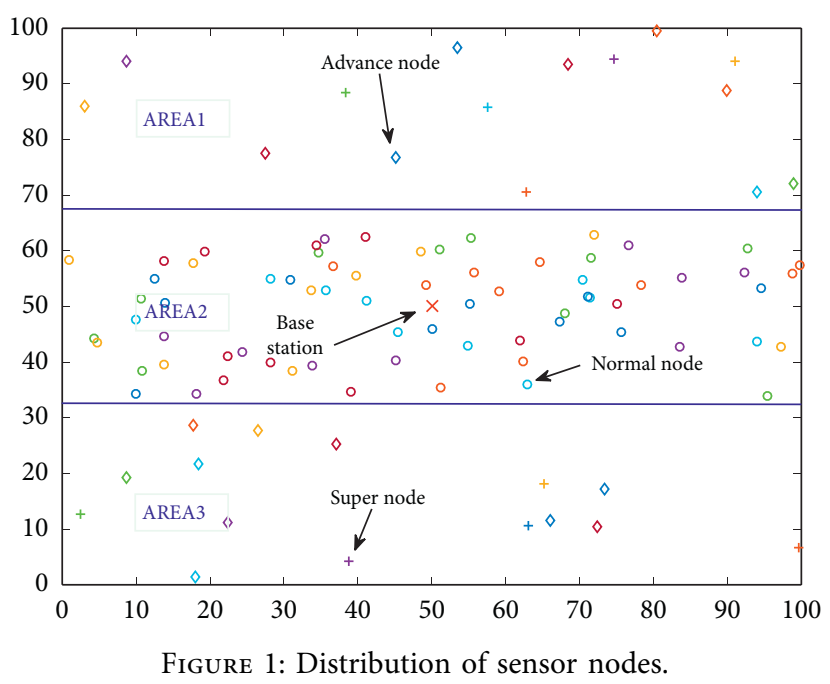

$$
\begin{aligned}
& T\left(s_{\text {nrm }}\right)= \begin{cases}\frac{p_{\text {nrm }}}{1-p_{\text {nrm }}\left[r \times \bmod \left(1 / p_{\text {nrm }}\right)\right]} \times \frac{E_{\text {res }}(r)}{E_{\text {init-nrm }},} & \text { if } s \in G_{1}, \\
0, & \text { if } s \notin G_{1},\end{cases} \\
& T\left(s_{\text {adv }}\right)= \begin{cases}\frac{p_{\text {adv }}}{1-p_{\text {adv }}\left[r \times \bmod \left(1 / p_{\text {adv }}\right)\right]} \times \frac{E_{\text {res }}(r)}{E_{\text {init-adv }},} & \text { if } s \in G_{2}, \\
0, & \text { if } s \notin G_{2},\end{cases} \\
& T\left(s_{\text {sup }}\right)= \begin{cases}\frac{p_{\text {sup }}}{1-p_{\text {sup }}\left[r \times \bmod \left(1 / p_{\text {sup }}\right)\right]} \times \frac{E_{\text {res }}(r)}{E_{\text {init-sup }},} & \text { if } s \in G_{3}, \\
0, & \text { if } s \notin G_{3},\end{cases}
\end{aligned}
$$

where $p_{\text {nrm }}, p_{\text {adv }}$, and $p_{\text {sup }}$ are the optimal probability of normal nodes, advance nodes, and supernodes to become a $\mathrm{CH}$, respectively, while $G_{1}, G_{2}$, and $G_{3}$ are sets of normal nodes, advance nodes, and supernodes that have not been selected as $\mathrm{CHs}$, respectively. The $E_{\text {res }}(r)$ is the residual energy of node at $r$ round, $E_{\text {init-nrm }}, E_{\text {init-adv }}$, and $E_{\text {init-sup }}$ are the initial energy of normal nodes, advance nodes, and supernodes, respectively.

Area 2 contained numerous normal nodes and was dense and likely to cause transmission interference, data redundancy, and collision. This would increase energy consumption and affect the efficiency of energy limited WSN. We will adopt the following $\mathrm{CH}$ election method as follows.

In Area 2, the normal node selects a node paired with itself within the communication range according to the neighbor node information table. If a node fails to find a neighbor node and becomes an isolated node, this occurred because the minimum distance between the isolated node and other nodes was outside its range. We then set half of the pairs of nodes to be active and set the other half of the pairs of nodes to be asleep at the current round. The isolate nodes are set to be active all of the time. The active nodes are selected as $\mathrm{CH}$ according to equation (5).
After the nodes are clustered, all nodes send the label, the remaining energy, and the perceived environment parameters to $\mathrm{CHs}$. CHs aggregate the information into data packets and wait for the information from the BS. The information includes paths through which $\mathrm{CHs}$ will transmit the data to the BS.

By establishing a new network model, low-energy nodes save energy during data transmission. Furthermore, by improving the threshold formula, nodes with large residual energy will have a greater probability of electing $\mathrm{CHs}$.

4.2. Data Transmission Phase. In the TSEP algorithm, the information will be directly transmitted from the cluster head to the base station. If the distance between the cluster head and base station is longer, it will consume much energy of the cluster head. This reduces the lifetime of the cluster head. In our improved protocol, we will adopt two schemes for data transmission. The $\mathrm{CHs}$ of Area 1 and Area 3 directly transmit information to the BS. However, the node energy of Area 2 is less than that of Area 1 and Area 3. Therefore, the $\mathrm{CH}$ of Area 2 will adopt a combination method of multihop and single-hop to transmit 
information to the BS [21]. The transmission mode of Area 2 is described in detail below.

The BS according to the node information table calculates the $\mathrm{CH} i$ communication energy consumption during the transmission of $l$ bit messages directly to the BS, which is defined as $E_{C i-B S}$. The value of $E_{C i-B S}$ is given by the following equation:

$$
E_{\mathrm{C} i-\mathrm{BS}}\left(l, d_{\mathrm{C} i-\mathrm{BS}}\right)= \begin{cases}l E_{\mathrm{elec}}+l \varepsilon_{\mathrm{fs}} d_{C i-\mathrm{BS}}^{2}, & d_{\mathrm{C} i-\mathrm{BS}}<d o, \\ l E_{\mathrm{elec}}+l \varepsilon_{\mathrm{mp}} d_{C i-\mathrm{BS}}^{4}, & d_{C i-\mathrm{BS}} \geq d o,\end{cases}
$$

where $E_{\text {elec }}$ expresses the consumed energy that one sensor node receives or transmits 1-bit information. The parameters $\varepsilon_{\mathrm{fs}}$ and $\varepsilon_{\mathrm{mp}}$ are used to express the consumed energy that signal amplifier processing 1-bit information in free-space propagation model and multipath fading model, respectively. $d_{C i-\mathrm{BS}}$ is used to express the distance between sensor node $i$ and base station. It can be expressed as follows:

$$
d_{C i-\mathrm{BS}}=\sqrt{\left(x_{C i}-x_{\mathrm{BS}}\right)^{2}+\left(y_{\mathrm{Ci}}-y_{\mathrm{BS}}\right)^{2}},
$$

where $\left(x_{C i}, y_{C i}\right)$ is the location coordinate of sensor node $i$, the $\left(x_{\mathrm{BS}}, y_{\mathrm{BS}}\right)$ is the location coordinate of the base station. The parameter do is just a threshold. It can be expressed as follows:

$$
d o=\sqrt{\varepsilon_{\mathrm{fs}} / \varepsilon_{\mathrm{mp}}} .
$$

Based on (8)-(10), we can obtain the $E_{C i-B S}$. If formula (11) can be satisfied, the cluster head node $j$ will be used as an intermediate sensor node between the cluster head node I and base station. If the following equation is not be satisfied, the cluster head node $i$ will directly transmit data to the base station without using the intermediate cluster head.

$$
E_{C i-\mathrm{BS}}\left(l, d_{C i-\mathrm{BS}}\right) \geq E\left(l, d_{C i-\mathrm{C} j}\right)+E\left(l, d_{C j-\mathrm{BS}}\right),
$$

where $d_{C j-\mathrm{BS}}$ expresses the distance between the base station and cluster head node $j, d_{C i-C j}$ is the distance between the cluster head node $i$ and the cluster head node $j$. The expressions of $d_{C i-C j}$ and $d_{C j-\mathrm{BS}}$ are given by the following equation:

$$
\begin{aligned}
& d_{C i-C j}=\sqrt{\left(x_{C i}-x_{C j}\right)^{2}+\left(y_{C i}-y_{C j}\right)^{2}}, \\
& d_{C j-\mathrm{BS}}=\sqrt{\left(x_{C j}-x_{\mathrm{BS}}\right)^{2}+\left(y_{C j}-y_{\mathrm{BS}}\right)^{2}} .
\end{aligned}
$$

This method can reduce the consumed energy that the cluster head transmits data to the base station. Suppose the distance between the cluster head and base station, and the cluster head will transmit data to the intermediate cluster head. In that case, the intermediate cluster head node transmits the received data to the base station.

After the cluster head node completes the data transmission, the cluster head will change its state. If the sensor node and its neighbor sensor node are not dead, then the two nodes should keep their current state. If a pair of sensor nodes have one dead node, its partner would keep an active status and try to find another node to complete a pair. After receiving the information, the $\mathrm{BS}$ updates the node information table and broadcasts the information to all nodes, and all nodes update the neighbor node information table according to the received information. This indicates the completion of this round. The proposed algorithm flow chart is shown in Figure 2.

\section{Simulation and Discussion}

5.1. Experiments. The experiment assumes that there are 100 nodes, $10 \%$ of the nodes are supernodes, $20 \%$ of the nodes are advanced nodes, and the remaining nodes are normal nodes. Each of the advanced nodes has 50\% more initialized energy than each of the normal nodes, and each of the supernodes has $100 \%$ more initialized energy than each of the normal nodes. The experiment assumes that each supernode has 1 Joules energy, so each advanced node and normal node have energy of 0.75 Joules and 0.5 Joules, respectively. The experiment randomly places these nodes in a square area of $100^{*} 100 \mathrm{~m}^{2}$, and the BS is located at the center. The best probability that each node becomes a $\mathrm{CH}$ is set to 0.1. Since the TSEP algorithm is based on the SEP algorithm, our proposed algorithm is also based on the TSEP algorithm. So this experiment uses the TSEP algorithm and SEP algorithm to compare the performance of dead nodes, network residual energy, and throughput with our proposed algorithm using the MATLAB platform as a simulation tool. The parameters required for the experiment are shown in Table 1.

Firstly, the sensor nodes are distributed in three zones that are shown in Figure 1. It can be used in monitoring the water quality of the fish pond. The normal sensor nodes are distributed in the center of the fish pond. The advance and supernodes are distributed at the edge of the pond. It is convenient to change the battery of sensor nodes that are distributed at the edge of the pond. Besides, if all sensor nodes have the same initial energy, the network can become a heterogeneous wireless sensor network after changing the battery of sensor nodes that are distributed at the edge of the pond. Figure 3 shows the percentage of death nodes per round. Figure 4 shows the residual energy of all nodes for SEP, TSEP, and the proposed algorithm. Figure 5 displays the number of packets that are between the $\mathrm{BS}$ and the $\mathrm{CHs}$ with respect to the number of rounds.

Secondly, the sensor nodes are distributed in a single zone. The percentage of death nodes in a single zone is shown in Figure 6. The residual energies of all nodes for SEP, TSEP, and proposed algorithm are shown in Figure 7. The throughputs of the three methods are shown in Figure 8.

5.2. Results Analysis. In Figure 3, it can be seen that the nodes start to die at about 1200 rounds when using SEP and TSEP, but they start to die at 1700 rounds when using the proposed method. The nodes completely died at about 2000, 2500 , and 4500 rounds when using SEP, TSEP, and our 


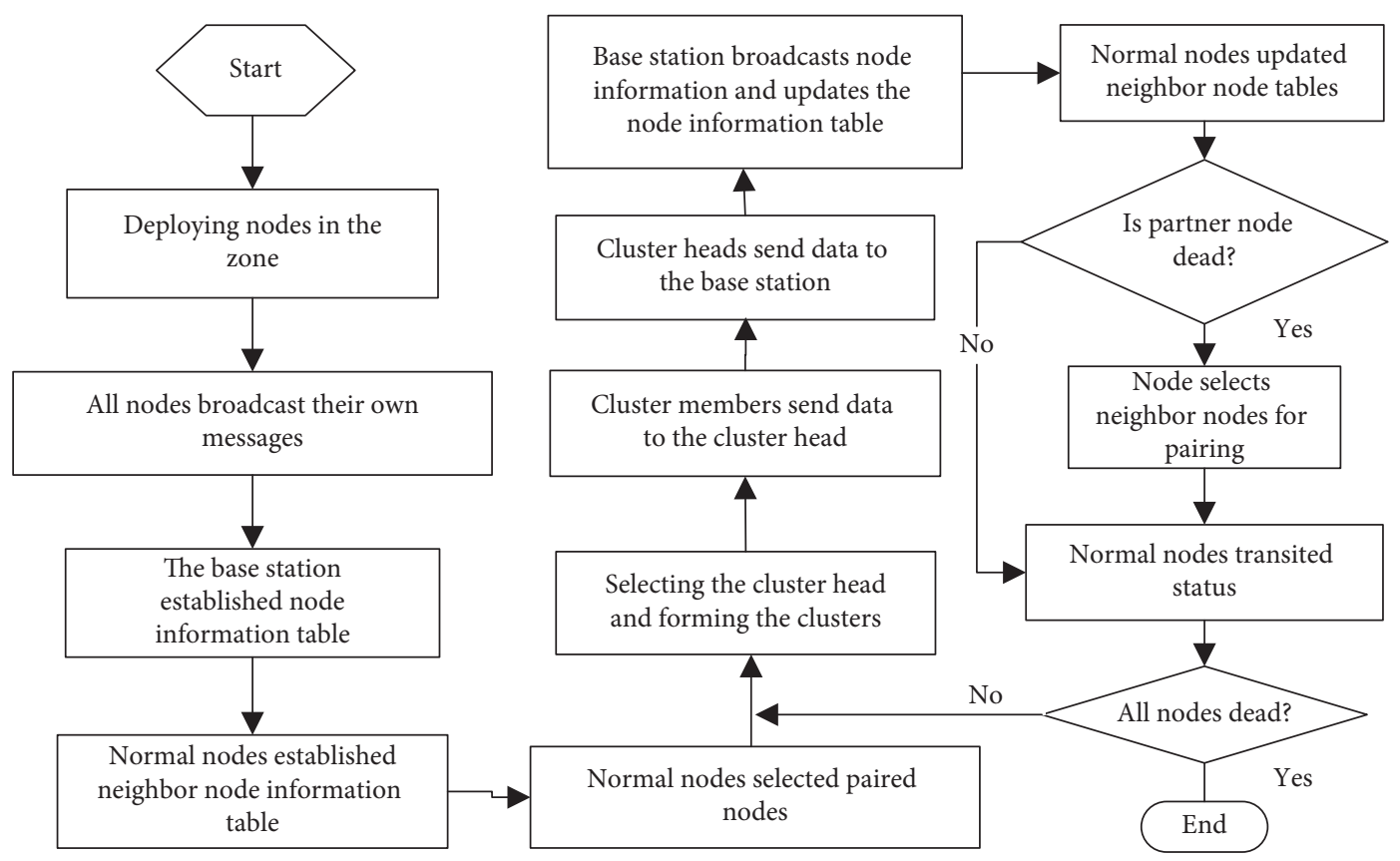

FIGURE 2: Flow chart of our proposed algorithm.

TABLE 1: The simulation parameters of the experiment.

\begin{tabular}{lcccccc}
\hline Parameters & $d o$ & $\varepsilon_{\mathrm{fs}}$ & $\varepsilon_{\mathrm{mp}}$ & $E_{\text {elec }}$ & $E_{\mathrm{DA}}$ & $l$ \\
\hline Values & 87 & 10 & 0.0013 & 50 & 5 & 4000 \\
\hline
\end{tabular}

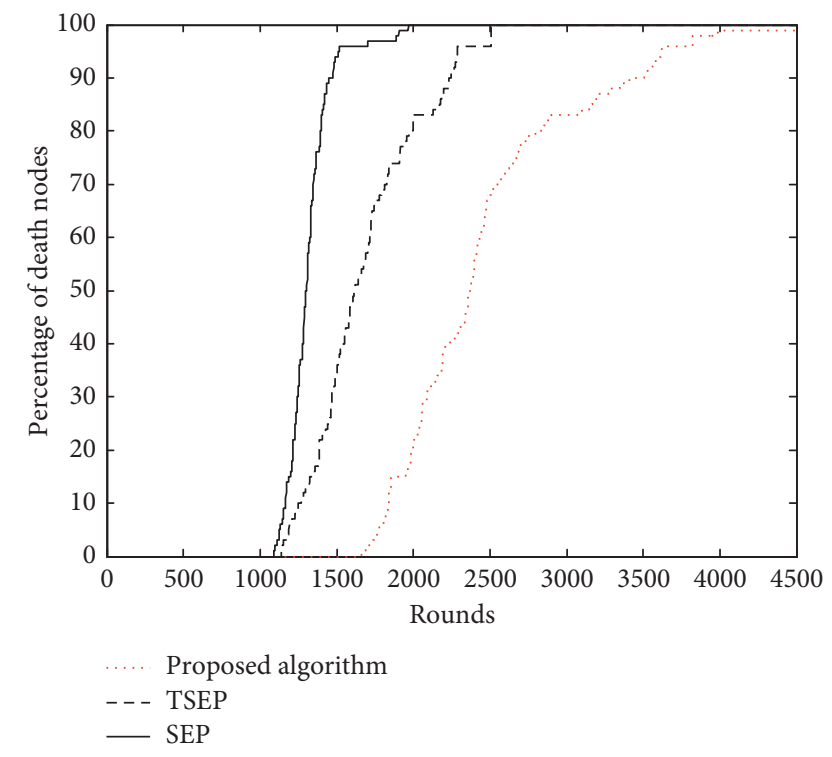

Figure 3: The percentage of death nodes with tree zones.

proposed method, respectively. There still are about 30 surviving nodes for the proposed method at 2500 rounds.

In Figure 4, we can see that the residual energy decreases with the increasing of rounds for all methods, but the proposed method has more residual energy than the others. This enhancement is due to the improved protocol in the mechanism of $\mathrm{CH}$ selection and routing. By improving the

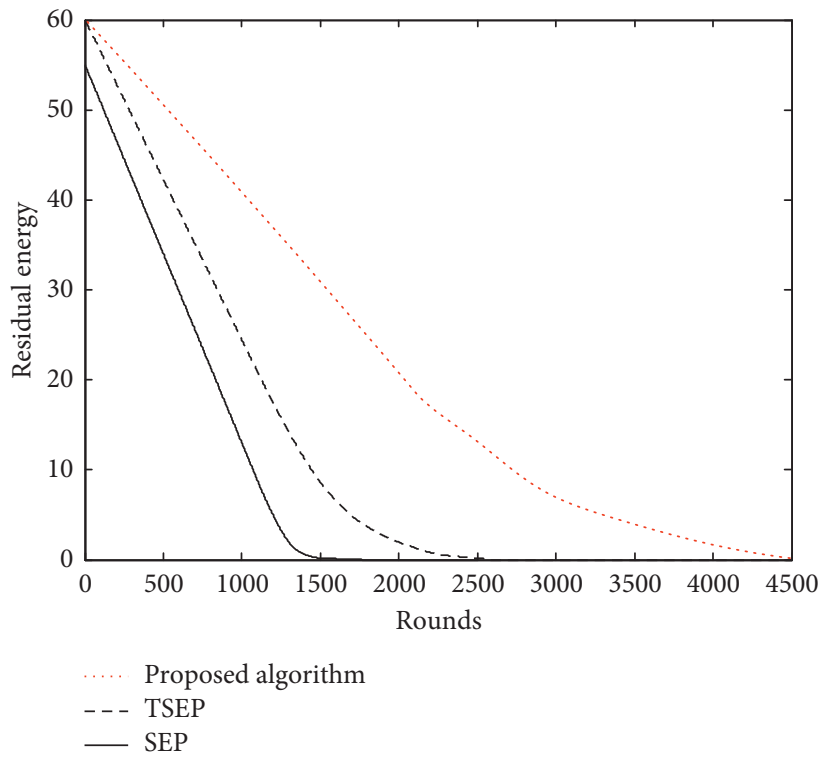

Figure 4: Residual energy with tree zones.

threshold, nodes with large residual energy have a greater possibility of becoming CHs to prevent premature death of low-energy nodes due to excessive energy consumption. In SEP and TSEP, the CH directly transmits data to the BS, but the proposed protocol adopts single hop and multiple hops to minimize the energy of the communication from $\mathrm{CH}$ to BS. Therefore, this proves that the proposed method prolongs the network's lifetime.

If there is a higher number of surviving nodes, there will be a higher number of selected $\mathrm{CHs}$, which means the proposed method prolongs the network's lifetime. The state transition mechanism in Area 2 can also successfully address 


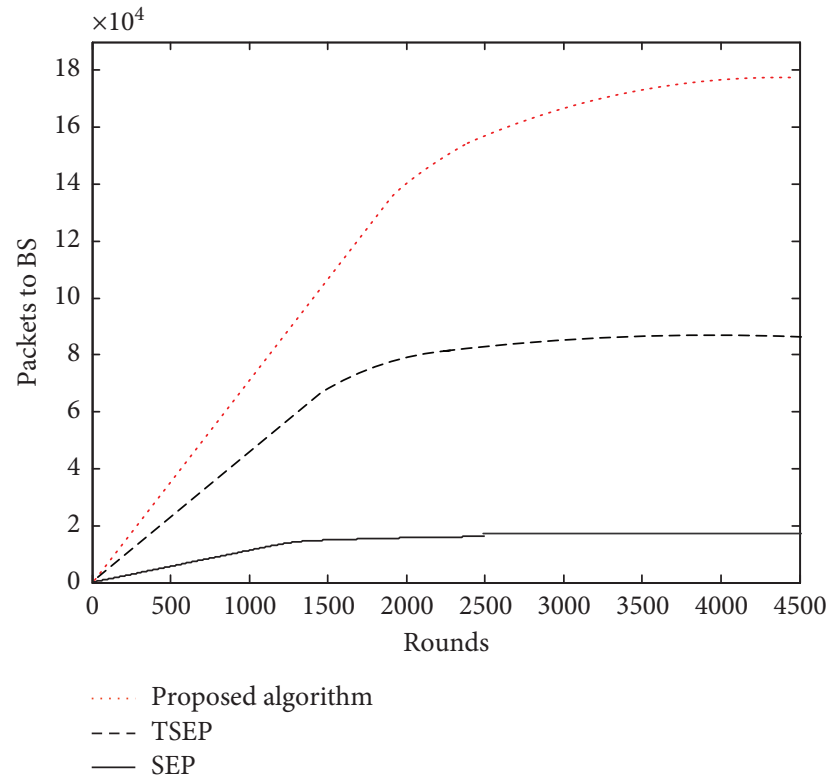

FIgURE 5: Throughput with tree zones.

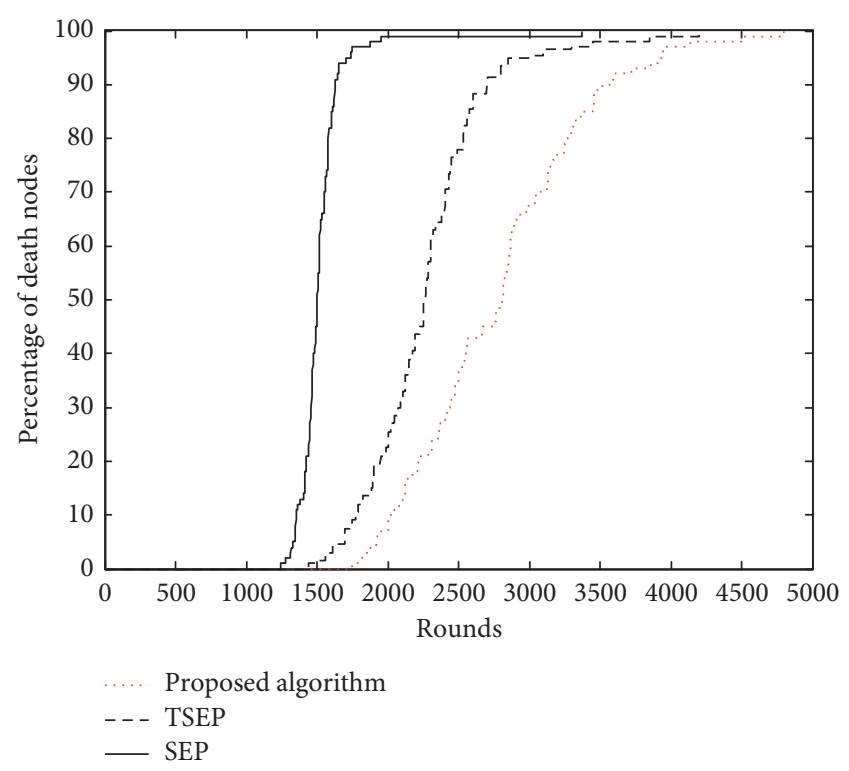

FIgURE 6: The percentage of death nodes with a single zone.

redundancy problems and the data conflict in TSEP and SEP. In Figure 5, the throughput of SEP and TSEP is less than our proposed method because only the cluster head sends data to the base station. In our proposed method, the data of the normal sensor node is directly sent to the base station, and the data of super and advanced sensor nodes that have more energy is sent to the base station by cluster head. Besides, the proposed method has a longer network lifetime than others. It has a longer time to transmit data.

In Figure 6, the nodes start to die at about 1300, 14000, and 1800 rounds when using SEP, TSEP, and our proposed method, respectively. Besides, The nodes completely died at about 3000, 4000, and 5000 rounds when using SEP, TSEP, and our proposed method, respectively. In Figure 7, the

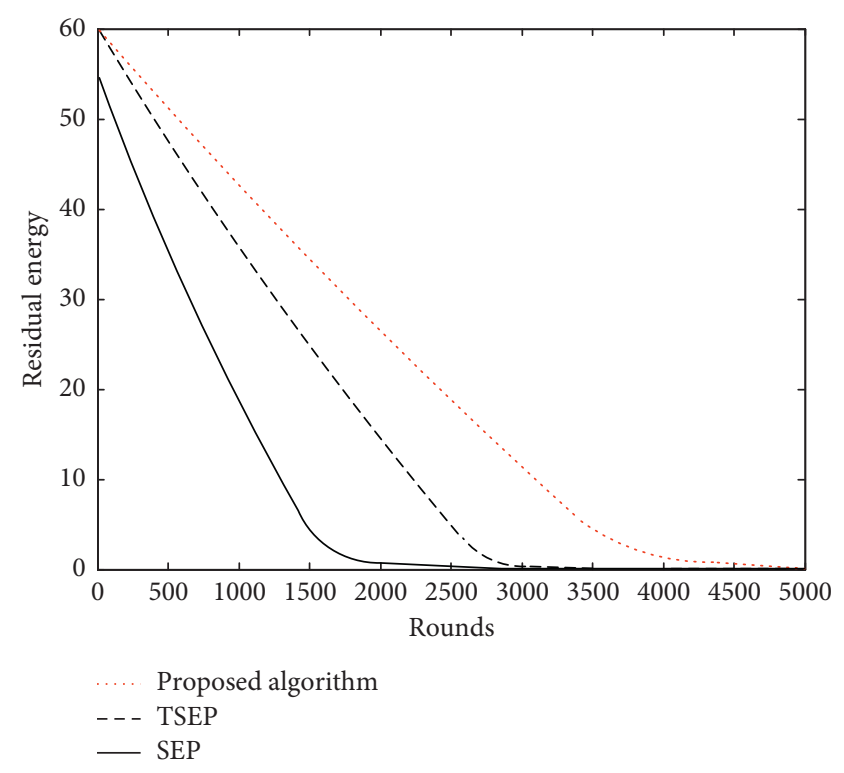

Figure 7: Residual energy with a single zone.

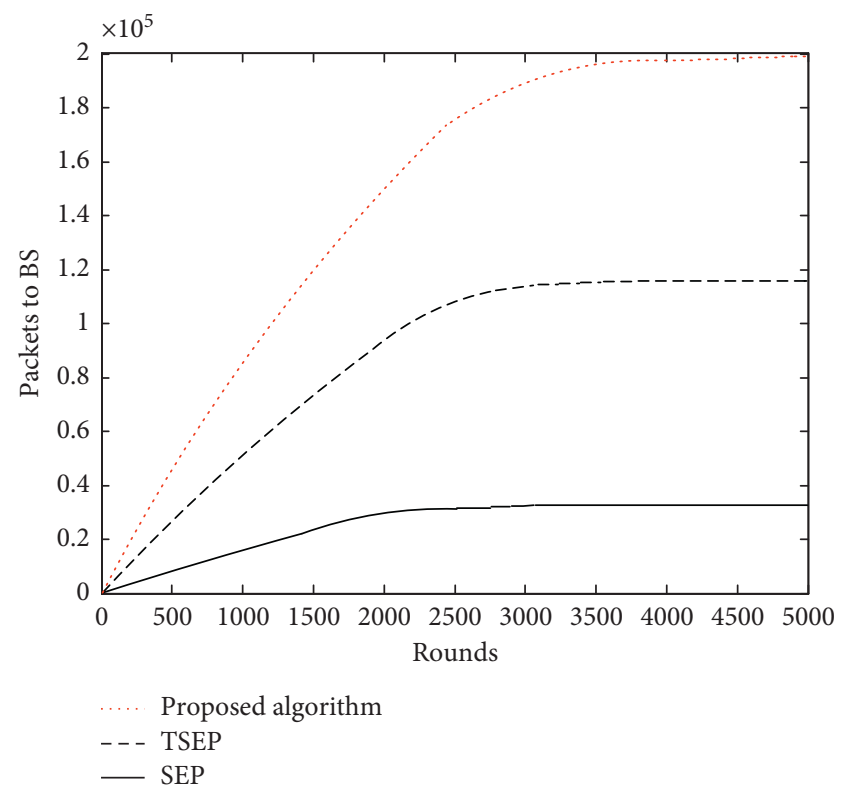

Figure 8: Throughput with a single zone.

residual energies reach zero at about 2000, 3000, and 4500 rounds for SEP, TSEP, and our proposed method, respectively. In Figure 8, we can also see that the number of packets for the proposed method is more than that of the other methods, and its lifetime is also longer than the other protocols. Based on the above analysis, this shows that the proposed method has better performance in prolonging the lifetime of the network for the sensor nodes that are distributed in tree zones and single zones.

\section{Conclusions}

An improved method has been designed to reduce the energy consumption and prolong network lifetime for three 
levels of energy HWSN. We divided the sensing area into three regions and deployed the supernodes, advanced nodes, and normal nodes separately. At the setup phase, it adjusts the threshold to select the nodes that have higher residual energy, which solves the problem of improper $\mathrm{CH}$ selection. It also uses a node state transformation mechanism to control the number of $\mathrm{CHs}$ in high-density node areas in Area 2. At the data transmission phase, it selects the path that consumes the minimum amount of energy as the optimal routing path to further reduce energy consumption. In comparison with the other methods, the proposed method prolongs the network lifetime.

In the proposed method, we supposed that the normal sensor node directly sends data to the base station. If the number of normal nodes is small or the space is large, it will affect the lifetime of the network. Besides, the proposed method increased time complexity, so this will affect the real-time of the network. The algorithm ignores the hotspot problem caused by the multihop transmission of data packets. The data loss problem and time complexity also should be research in the feature. This paper supposed that the sensor nodes cannot be moved, how to prolong the lifetime of movable sensor nodes should also be research in the feature.

\section{Data Availability}

The data used to support the findings of this study are available from the corresponding author upon request.

\section{Conflicts of Interest}

The authors declare that they have no conflicts of interest.

\section{Acknowledgments}

This work was supported by the National Natural Science Foundation of China (61901007), Scientific and Technological Developing Scheme of Jilin Province (YDZJ202101ZYTS172), Research Foundation of Education Bureau of Jilin Province (JJKH20210042KJ, JJKH20210095KJ), Doctoral Scientific Research Foundation of Beihua University (20171424), and Scientific Research Foundation of Jilin City (20190104124).

\section{References}

[1] P. K. Batra and K. Kant, "An energy efficient GA based routing algorithm for two-tiered sensor networks," in Proceedings of 2016 1st India International Conference on Information Processing (IICIP), pp. 1-6, Delhi, India, August 2016.

[2] R. Thapa, H. Singh, and A. Sharma, "A comparative analysis of LEACH and SEP using NS2," in Proceedings of 2017 8th International Conference on Computing, Communication and Networking Technologies (ICCCNT), pp. 1-4, Delhi, India, July 2017.

[3] H. El Alami and A. Najid, "ECH: an enhanced clustering hierarchy approach to maximize lifetime of wireless sensor networks," IEEE Access, vol. 7, pp. 107142-107153, 2019.

[4] J.-S. Lee and W.-L. Cheng, "Fuzzy-logic-based clustering approach for wireless sensor networks using energy predication," IEEE Sensors Journal, vol. 12, no. 9, pp. 2891-2897, 2012.

[5] H. El Alami and A. Najid, "MS-routing-G i: routing technique to minimise energy consumption and packet loss in WSNs with mobile sink," IET Networks, vol. 7, no. 6, pp. 422-428, 2018.

[6] A. Kashaf, N. Javaid, and Z. A. Khan, "TSEP: thresholdsensitive stable election protocol for WSNs," in Proceedings of 2012 International Conference on Frontiers of Information Technology, pp. 164-168, Islamabad, Pakistan, December 2012.

[7] G. Smaragdakis, I. Matta, and A. S. E. P. Bestavros, "A stable election protocol for clustered heterogeneous wireless sensor networks," 2019, https://open.bu.edu/ds2/stream/? \#/documents/4866/page/1.

[8] L. Qing, Q. Zhu, and M. Wang, "Design of a distributed energy-efficient clustering algorithm for heterogeneous wireless sensor networks," Computer Communications, vol. 29, no. 12, pp. 2230-2237, 2006.

[9] P. Azad, B. Singh, and V. Sharma, "A novel clustering approach for extending the lifetime for wireless sensor networks," International Journal of Advances in Engineering and Technology, vol. 1, pp. 441-446, 2011.

[10] O. Rehman, N. Javaid, B. Manzoor, A. Hafeez, A. Iqbal, and M. Ishfaq, "Energy consumption rate based stable election protocol (ECRSEP) for WSNs," Procedia Computer Science, vol. 19, pp. 932-937, 2013.

[11] V. Srividhya and T. Shankar, "Energy proficient clustering technique for lifetime enhancement of cognitive radio-based heterogeneous wireless sensor network," International Journal of Distributed Sensor Networks, vol. 14, Article ID 1876759, , 2018.

[12] S. Faisal, N. Javaid, A. Javaid, M. A. Khan, S. H. Bouk, and Z. A. Khan, "Z-SEP: Zonal-stable election protocol for wireless sensor networks," Journal of Research in Basic and Applied Science, vol. 3, pp. 132-139, 2012.

[13] Y. K. Tamandani and M. U. Bokhari, "SEPFL routing protocol based on fuzzy logic control to extend the lifetime and throughput of the wireless sensor network," Wireless Networks, vol. 22, no. 2, pp. 647-653, 2016.

[14] T. Jinpa and B. R. Reddy, M-SEP: A Variant of SEP for WSN. Intelligent Systems Technologies and Applications, MDPI, Basel, Switzerland, 2016.

[15] J. Peng, T. Liu, H. Y. Li, and B. Guo, "Energy-efficient prediction clustering algorithm for multilevel heterogeneous wireless sensor networks," International Journal of Distributed Sensor Networks, vol. 2013, Article ID 678214, 8 pages, 2013.

[16] Z. Hong, L. U. Yu, and G. J. Zhang, "Efficient and dynamic clustering scheme for heterogeneous multilevel wireless sensor networks," Acta Automatica Sinica, vol. 39, pp. 454460, 2016

[17] S. Hassan, M. Nisar, and H. Jiang, "Energy preservation in heterogeneous wireless sensor networks through zone partitioning," Indonesian Journal of Electrical Engineering and Computer Science, vol. 2, no. 2, pp. 390-395, 2016.

[18] J.-S. Leu, T.-H. Chiang, M.-C. Yu, and K.-W. Su, "Energy efficient clustering scheme for prolonging the lifetime of wireless sensor network with isolated nodes," IEEE Communications Letters, vol. 19, no. 2, pp. 259-262, 2015.

[19] J.-S. Lee and C.-L. Teng, "An enhanced hierarchical clustering approach for mobile sensor networks using fuzzy inference systems," IEEE Internet of Things Journal, vol. 4, no. 4, pp. 1095-1103, 2017. 
[20] H. El Alami and N. Abdellah, "Energy-efficient fuzzy logic cluster head selection in wireless sensor networks," in Proceedings of the 2016 International Conference on Information Technology for Organizations Development (IT4OD), IEEE, Fez, Morocco, April 2016.

[21] L. Zhao and Q. Tang, "An improved threshold-sensitive stable election routing energy protocol for heterogeneous wireless sensor networks," Information, vol. 10, no. 4, p. 125, 2019. 